\title{
Insulin, but Not Metformin, Supports Wound Healing Process in Rats with Streptozotocin-Induced
} Diabetes

This article was published in the following Dove Press journal: Diabetes, Metabolic Syndrome and Obesity: Targets and Therapy

\section{Mateusz Mieczkowski (iD) \\ Beata Mrozikiewicz- \\ Rakowska (D) \\ Tomasz Siwko' \\ Magdalena Bujalska- \\ Zadrozny (D) ${ }^{2}$ \\ Anna de Corde-Skurska ${ }^{2}$ \\ Renata Wolinska (iD) ${ }^{2}$ \\ Emilia Gasinska ${ }^{2}$ \\ Tomasz Grzela $\left(\mathbb{D}^{3}\right.$ \\ Piotr Foltynski (iD) \\ Michal Kowara ${ }^{5}$ \\ Zofia Mieczkowska' \\ Leszek Czupryniak (D)}

'Department of Diabetology and Internal Medicine, Medical University of Warsaw, Warsaw, Poland; ${ }^{2}$ Department of

Pharmacodynamics, Medical University of

Warsaw, Warsaw, Poland; ${ }^{3}$ Department

of Histology and Embryology, Medical

University of Warsaw, Warsaw, Poland;

${ }^{4} \mathrm{Nalecz}$ Institute of Biocybernetics and

Biomedical Engineering Polish Academy

of Sciences, Warsaw, Poland;

${ }^{5}$ Department of Cardiology, Medical

University of Warsaw, Warsaw, Poland

Correspondence: Beata Mrozikiewicz-

Rakowska

Department of Diabetology and Internal

Medicine, Medical University of Warsaw,

Poland ul. Banacha IA, Warsaw, 02-097,

Poland

Tel +486003II 399

$\mathrm{Fax}+48225992832$

Email rakowskabI23@gmail.com
Purpose: Optimal glycemic control is crucial for proper wound healing in patients with diabetes. However, it is not clear whether other antidiabetic drugs support wound healing in mechanisms different from the normalization of blood glucose control. We assessed the effect of insulin and metformin administration on the wound healing process in rats with streptozotocin-induced diabetes.

Methods: The study was conducted on 200 male Wistar rats with streptozotocin-induced diabetes. In the last phase of the study, 45 rats, with the most stable glucose levels in the range of $350-500 \mathrm{mg} / \mathrm{dL}$, were divided into three groups: group I received human nonprotamine insulin subcutaneously ( $5 \mathrm{IU} / \mathrm{kg}$ body mass) once a day, group II received metformin intragastrically (500 $\mathrm{mg} / \mathrm{kg}$ b.m.), and group III (control) was given saline subcutaneously. After 14 days of antidiabetic treatment, a $2 \mathrm{~cm} \times 2 \mathrm{~cm}$ thin layer of skin was cut from each rat's dorsum and a $4 \mathrm{~cm}$ disk with a hole in its center was sewn in to stabilize the skin and standardize the healing process. The wound healing process was followed up for 9 days, with assessment every 3 days. Biopsy samples were subjected to hematoxylin and eosin staining and immunohistochemical assays.

Results: Analysis of variance revealed significant influence of treatment type (insulin, control, or metformin) on the relative change in wound surface area. The wound healing process in rats treated with insulin was more effective than in the metformin and control groups. Wound tissue samples taken from the insulin-treated animals presented significantly lower levels of inflammatory infiltration. Immunohistochemical assessment showed the greatest density of centers of proliferation $\mathrm{Ki}-67$ in insulin-treated animals.

Conclusion: These results suggest that an insulin-based treatment is more beneficial than metformin, in terms of accelerating the wound healing process in an animal model of streptozocin-induced diabetes.

Keywords: diabetes mellitus, neuropathy, ulceration, animal model

\section{Introduction}

The rapidly growing incidence of diabetes is accompanied by a simultaneous increase in the number of complications caused by this disease. A particular example of such a complication is disordered wound healing. One of the most common wounds in diabetes is diabetic foot syndrome (DFS). ${ }^{1}$ Estimates show that up to $30 \%$ of people with diabetes may develop DFS. The prevalence of DFS in a diabetic patient is $19 \%$ $34 \%$, with an annual incidence of $2 \%$. After the healing process is completed, the rate of ulcer recurrence is $40 \%$ per year, with $65 \%$ recurrence within 3 years. It should be 
emphasized, however, that only two-thirds of patients with DFS are likely to heal, and about $28 \%$ of patients will require lower limb amputation. ${ }^{2-4}$ The socioeconomic cost of care for DFS has become a global economic problem, especially taking into consideration the fact that one amputation of the lower limb is carried out every $20 \mathrm{~s}$ owing to this disease. ${ }^{5,6}$

For this reason, in the modern development of the pharmacotherapy of diabetes and its complications, it is important to emphasize not only the control of diabetes itself, but also activities that can prevent or inhibit the development of complications, eg, wounds induced by the presence of chronic hyperglycemia. A good example is the use of the pleiotropic effect of sodium-glucose cotransporter-2 (SGLT-2) inhibitors, which have a range of actions other than their hypoglycemic effect, eg, they inhibit the progression of heart failure. ${ }^{7}$

The prolonged wound healing observed in diabetes results from the different pathophysiology of the wound healing process under hyperglycemia. It has been proven that, under conditions of hyperglycemia, the wound healing process is significantly impaired. Christman et al showed that for each percentage point increase in the level of glycated hemoglobin (HbA1c), the daily woundarea healing rate was decreased by $0.028 \mathrm{~cm} 2$ per day in humans. $^{8}$ Hyperglycemia affects the persistence of a chronic inflammatory process in the wound, often leading to infectious etiologies. In addition, hyperglycemia significantly impairs the body's defense functions, which are dependent on cellular and humoral factors. Insulin deficiency, caused by insulin resistance in type 2 diabetes or its complete absence in type 1 diabetes, affects the activity of insulin-dependent enzymes contained in neutrophils. Myeloperoxidase and superoxide dismutase, contained in the azurophilic granules of neutrophils, show reduced activity, with weakened bactericidal properties. Deficiencies in nicotinamide adenine dinucleotide phosphate (NADPH), which is connected to the activation of the polyol pathway in diabetes, result in dysfunction of the oxygen mechanisms responsible for neutralization of microorganisms by neutrophils (including the formation of reactive oxygen species on the cell membrane). Monocyte- and macrophage-dependent chemotaxis and phagocytosis are also impaired. Perfusion disorders, leading to critical ischemia at both the micro- and macrovascular levels, underlie the pathophysiology of diabetes, and are direct factors in extending the duration of wound healing, often precluding a successful healing process. ${ }^{9-11}$
In addition to local treatment methods for chronic wounds (eg, vacuum-assisted closure or the use of growth factors), methods are being sought to optimize tissue regeneration on a systemic scale. It is postulated that there may be alternative mechanisms of action of antidiabetic drugs, which are independent of their effect on glycemia. These drugs might have a direct impact on the process of regenerating defective tissue in the treatment of ulcers.

The introduction of a more effective pharmacotherapy is made possible through a better understanding of mechanisms accompanying the development of wounds, ascertained through the studies using properly developed animal models. ${ }^{12-14}$ Assuming that the study of properly developed animal models that reflect the tissue regeneration processes as faithfully as possible in patients with long-term diabetes will allow the selection of more effective pharmacotherapy in future, we decided to compare the effect of insulin and metformin on the healing process of chronic wounds in an experimental model of streptozotocin-induced diabetes in Wistar rats.

\section{Materials and Methods}

The study was conducted after obtaining the consent of the 2nd Local Ethical Commission for Animal Experiments at the Medical University of Warsaw (Resolution No. 17/2014 of 25 February, 2014). In addition, we also include the name of the guidelines followed for the welfare of the laboratory animals of Polish National Ethical Committee for Animal Experiments. ${ }^{15}$ A total of 200 inbred male Wistar rats, weighing $300 \pm 30 \mathrm{~g}$ were used for the study (Figure 1). The laboratory part of the study consisted of five phases.

In the initial phase, 120 rats were used to determine the insulin demand and dosage of antidiabetic drugs; a further 80 rats were used in the core stage of the experiment. During the trial, animals had unlimited access to water and food.

\section{Phase I: Induction of Diabetes in Study Animals}

To induce diabetes, all subjects received streptozotocin (Sigma-Aldrich, St. Louis, MO, USA) intramuscularly at a dose of $35 \mathrm{mg} / \mathrm{kg}$ body mass (b.m.) ${ }^{16}$ The levels of glucose were determined using an Accu-chek Active ${ }^{\circledR}$ glucometer (Roche, Basel, Switzerland) four times a day. Blood samples for glucose determination were drawn from the tail vein. The development of diabetes was accompanied by the development of neuropathy. After 7 days, 


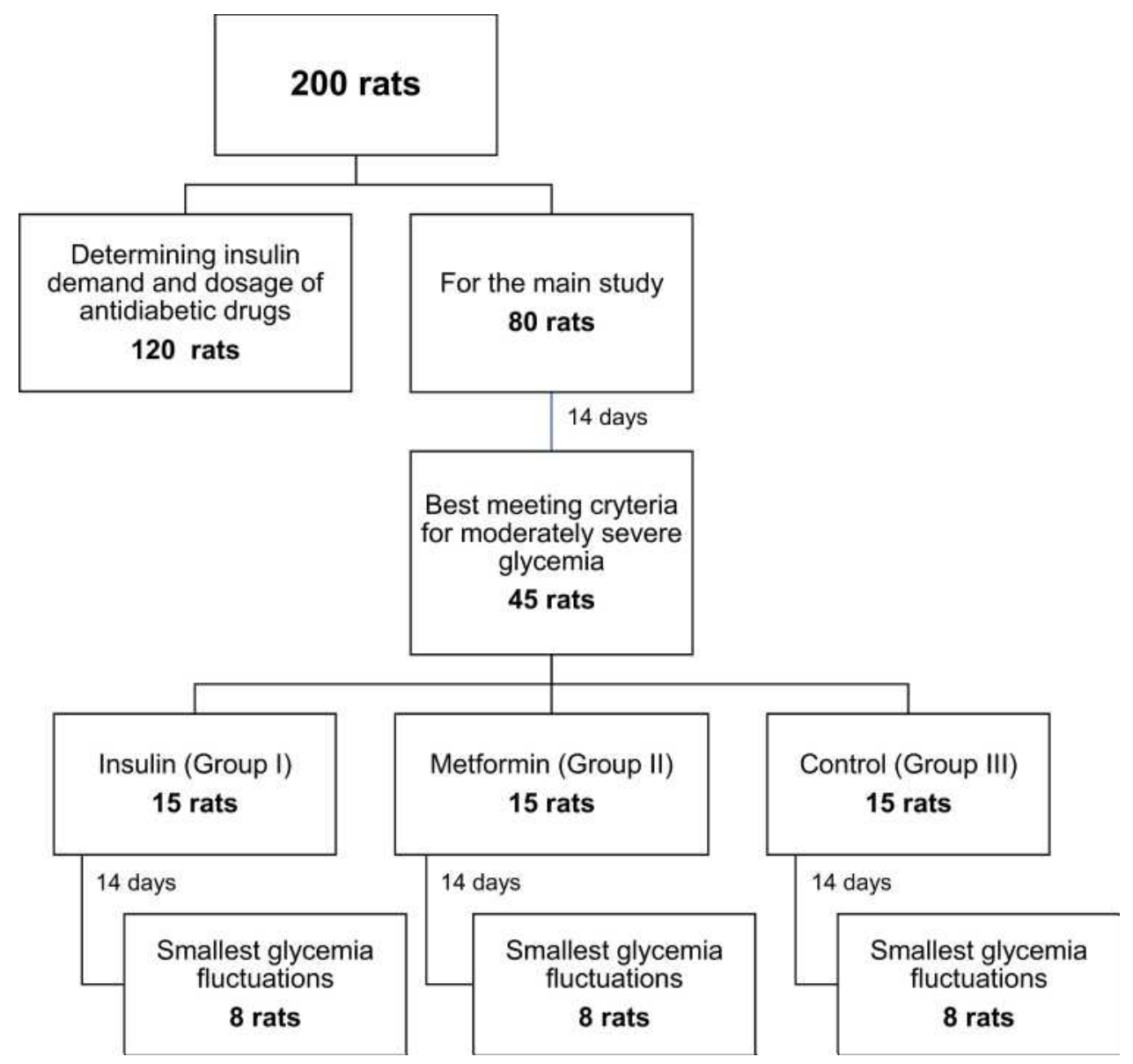

Figure I Study protocol.

sensory neuropathy was observed in the study animals. The degree of nerve damage was assessed at 7, 14, 21, and 28 days after streptozotocin administration by measuring the sensitivity threshold to a mechanical stimulus, using a modification of the Randall-Selitto test. ${ }^{17}$ Changes in the sensory threshold to a mechanical stimulus were studied using an Ugo-Basile analgesimeter type $7200^{\circledR}$ (Biological Research Apparatus, Comerio-Varese, Italy). A mechanical stimulus of increasing pressure, applied to the outer surface of the rat's hind limb, was delivered by the analgesimeter until the limb twitched or was withdrawn by the rat. The results, obtained in grams, were converted into percentage variations from the values obtained before streptozotocin administration (time 0 ) for each rat, according to the following formula:

$$
\% \text { analgesia }=\frac{(100 \times \mathrm{B})}{\mathrm{A}}-100
$$

where

$A=$ pressure at time 0 (prior to streptozotocin administration),
$B=$ pressure on subsequent days following streptozotocin administration.

The percentages of analgesia for individual animals, calculated according to the formula, were then used to calculate the average values for the groups (Table 1).

\section{Phase 2: Determining Required Insulin and Metformin Doses in Study Animals}

After the period of diabetes induction, the rats were divided into two groups. The first 120 individuals were used to determine the appropriate doses of drugs, ie, metformin and insulin. Initial drug doses were determined on the basis of data from the literature. ${ }^{18,19}$ Fourteen days after the administration of streptozotocin, from a second group, of 80 individuals, 45 rats were selected who best met the criteria for mild diabetes (blood glucose $>200 \mathrm{mg} / \mathrm{dL}$ ). These 45 rats were then divided into three groups: 15 subjects, group I, were treated with insulin (Insulatard ${ }^{\circledR}$, Novo Nordisk A/S Novo Allé, Bagsværd, Denmark) preparations of $5 \mathrm{IU} / \mathrm{kg}$ b.m. subcutaneously; 15 rats, group II, were treated with metformin 
Table I Percentage Changes in Pain Threshold in the Development of Neuropathy (Day 0 - Before Streptozotocin Administration, Day 7, Day 14, Day 21 and Day 28 After Streptozotocin Administration), Compared with Values Before Streptozotocin Administration (measured using a modification of the Randall-Selitto test) ${ }^{17}$

\begin{tabular}{|l|l|l|l|l|l|l|}
\hline \multirow{2}{*}{ Group } & \multicolumn{2}{l|}{ Insulin (Group I) } & \multicolumn{2}{l|}{ Metformin (Group II) } & \multicolumn{2}{l|}{ Control (Group III) } \\
\cline { 2 - 7 } & Antinociception & Reduction (\%) & Antinociception & Reduction (\%) & Antinociception & Reduction (\%) \\
\hline Before streptozotocin & 108.215 & & 118.125 & & 112.8125 & \\
administration & & & & & & \\
Day 7 & 95 & -11.80 & 94.375 & -19.85 & 94.375 & -16.28 \\
Day 14 & 66.5625 & -38.33 & 76.25 & -35.40 & 75.00 & -33.47 \\
Day 21 & 64.375 & -40.37 & 68.75 & -41.49 & 63.125 & -41.49 \\
Day 28 & 60.9375 & -43.60 & 66.5625 & -43.49 & 60.9375 & -44.00 \\
\hline
\end{tabular}

(Metformax ${ }^{\circledR}$, Teva Pharmaceuticals Polska sp.z.o.o., Warsaw, Poland) at a dose of $500 \mathrm{mg} / \mathrm{kg}$ b.m. orally; and the remaining 15 individuals, who formed the control group (group II), were administered $0.9 \% \mathrm{NaCl}$ at dose of $50 \mu \mathrm{L} / \mathrm{kg}$ b.m. subcutaneously.

\section{Phase 3: Wound Formation}

After 14 days of treatment, eight individuals with the smallest glucose fluctuations were selected from each group (ranges: group I, 326-488 mg/dL; group II, 340-511 mg/ dL; group III, 359-510 mg/dL). Scarification was made on the dorsal surface of the skin, after shaving the fur using an electric razor. All animals selected for this phase of the study had a piece of skin removed from the dorsum to the depth of the panniculus carnosus muscle, $0.3-0.5 \mathrm{~mm}$ thick and $2 \mathrm{~cm}$ $\times 2 \mathrm{~cm}$ wide. ${ }^{20-22}$ Before surgery, the animals were subjected to general anesthesia using a mixture of isoflurane (Isofluran Baxter $^{\mathbb{R}}$, Søborg, Denmark) and air. The induction of anesthesia was carried out in a sealed chamber at an isoflurane concentration of $4-5 \%$ and a flow of $1000 \mathrm{~mL} / \mathrm{min}$. Next, to maintain anesthesia, a mixture of 3-3.5\% isoflurane in air was administered via a mask outside the induction chamber (Isoflurane Vaporizer, Rothacher \& Partner, Berne, Switzerland). Before performing the surgery, the rats' reflexes were always checked by applying pressure using tweezers in the midfoot and the tail. In each group, $1 \mathrm{~mL}$ of lipopolysaccharide solution obtained from Pseudomonas aeruginosa (Sigma-Aldrich) at a concentration of $1 \mathrm{mg} / \mathrm{mL}$ was applied to the wounds to produce chronic inflammation. ${ }^{23}$ Then, using surgical sutures, a $4 \mathrm{~cm} \times$ $4 \mathrm{~cm}$ silicone plate with a hole in the center was sutured in place over the wound to replace the skin and standardize the wound healing process. Samples from the marginal sections of the healthy cut-out skin were also collected. Every 3 days, in each individual, a sample was collected from a marginal section of the wound, wound surface area was assessed, and the protective dressing changed (Mollelast ${ }^{\circledR} 4 \mathrm{~cm} \times$ $4 \mathrm{~m}$ band, Lohmann \& Rauscher, Pabianice, Poland). The protective dressing was gently put around the upper limbs and three-quarters of the upper torso. The bandage was cut in key places (eg, around the shoulder joint) with a risk of compression complications (ischemia, acute neuropathy), creating a kind of a sleeve. The ends of the band were secured with adhesive tape.

\section{Phase 4: Analysis of the Wound Healing Process}

To measure the wound surface area, the Planimator app (design by Institute of Biocybernetics and Biomedical Engineering, Polish Academy of Sciences), installed on a Samsung Galaxy S5 smartphone, was used. ${ }^{24}$ To measure the surface area with this app, two self-adhesive rulers were used, placed on opposite sides of the wound, and the wound contour was outlined in a photograph showing the wound and the rulers located around it.

\section{Phase 5: Histological Evaluation of Collected Sections}

Sections taken from wounds were fixed in $2 \%$ formaldehyde solution. Standard staining methods (hematoxylin and eosin) were used to determine the presence of inflammatory cells, and standard immunohistochemical testing was used to determine the presence of the nuclear proliferation marker Ki-67 antigen.

\section{Statistical Analysis}

The influence of treatment type on wound area was analyzed using analysis of variance (ANOVA). Post-hoc Scheffé and Bonferroni tests were also conducted. Normality of data distribution was analyzed using Kolmogorov-Smirnov and 
Shapiro-Wilk tests. Homogeneity of variances was determined using Bartlett's, Cochran's, Hartley's, and Levene's tests. Data were analyzed using STATISTICA ${ }^{\circledR}$ software version 10 (StatSoft Inc.,Tulsa, OK, USA). Statistical significance was considered for $p<0.05$.

\section{Results}

After administration of streptozotocin in the study rats, symptoms of increasing hyperglycemia were observed, up to maxima of $492 \mathrm{mg} / \mathrm{dL}$ in the insulin group (group I), $512 \mathrm{mg} / \mathrm{dL}$ in the metformin group (group II), and $526 \mathrm{mg} / \mathrm{dL}$ in the control group (group III). This was manifested by increased water intake, increased urine volumes, and reduced pain thresholds (Table 1).

During the 25 days from the start of drug administration, the average glucose level in each group stabilized, as shown in Figure 2. For the statistical analysis of glycemia results, the distribution of which was close to normal, ANOVA was used.

To avoid making the mistake of repeated testing, the comparison was made at an isolated point in time, specifically just prior to drug administration. The comparison of glucose concentration between the considered groups did not reveal any significant differences between the groups $(p=0.21)$; therefore, post hoc tests were not performed. In the subsequent days of the wound healing phase, the differences in glucose concentration between the insulin group (I) and the metformin group (II) were statistically insignificant, which makes it possible to treat glucose levels in these groups as equal. The results of the statistical analysis are shown in Table 2.
To monitor the wound healing process, changes in the wound surface area and macroscopic features of inflammation were assessed (Figure 3).

Percentage changes in wound surface area were calculated for each wound as the difference between the area at day 9 and the area at the start of treatment divided by the area at the start of treatment. The impact of treatment type on the change in wound area was analyzed using ANOVA. The result was significant $(p=0.002)$. The assumptions (homogeneity of variances and normality of data) for the ANOVA test were checked. There were no significant differences between variances $(U=0.31)$ and the percentage changes in wound area were normal for each treatment type (group I, $p=0.10$; group II, $p=0.43$; group III, $p=0.28)$. The mean changes in wound area ( \pm standard deviation) were for the insulin (group I), $-66.8 \%$ $( \pm 9.7 \%)$, metformin (group II) $-40.4 \%( \pm 15.8 \%)$, and control (group III) $-48.0 \%$ ( $\pm 9.8 \%$ ),respectively. Post-hoc Scheffé and Bonferroni tests revealed significant differences between the insulin (group I) and metformin (group II) groups, with $p<0.002$ for both tests and between the insulin (group I) and control (group III) groups, with $p<0.03$ for both tests. There were no significant differences between the metformin (group II) and control (group III) groups ( $p>0.51$ for both tests). Therefore, the highest rate of wound healing was for the insulin group (group I).

Histologically assessed wounds differed in the rate of resolution of inflammation. Biopsies taken on the day of wound formation (control biopsies) showed a normal histological picture of skin and subcutaneous tissue (Figure 4).

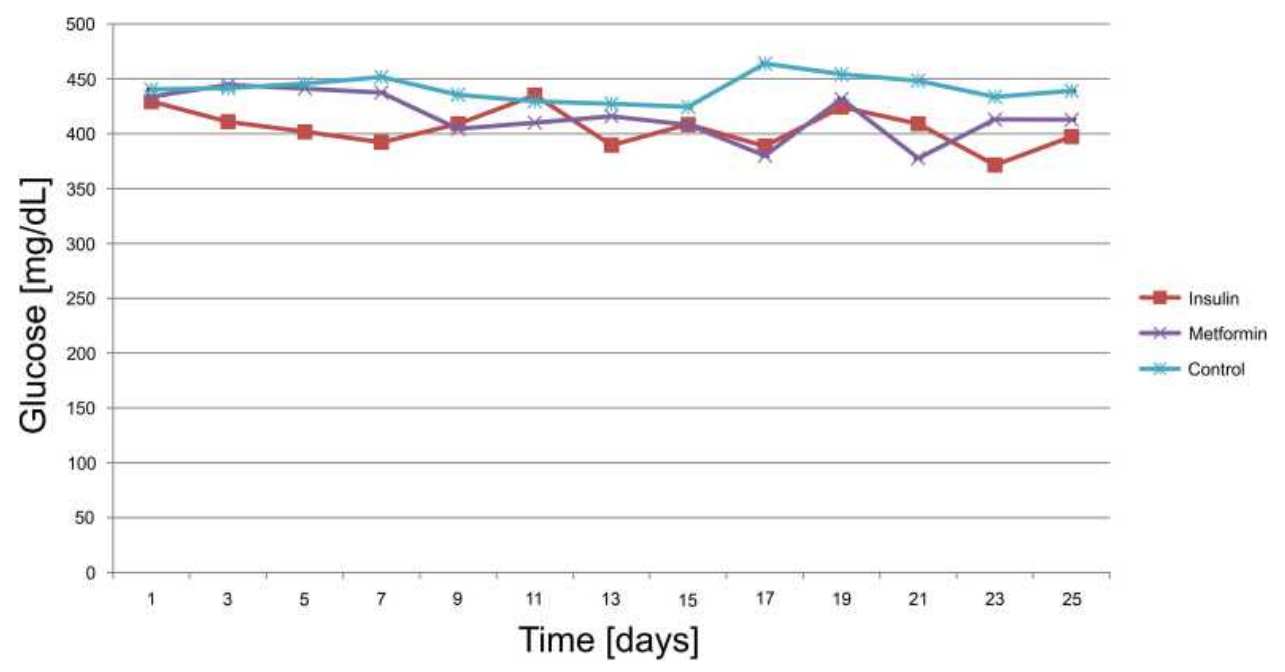

Figure 2 Glycemia in the analyzed groups during all phases of the study. 
Table 2 Results of Statistical Analysis for Glycemia in the Wound Healing Phase Between Groups (ANOVA)

\begin{tabular}{|c|c|c|c|c|}
\hline $\begin{array}{l}\text { Day After } \\
\text { Streptozotocin } \\
\text { Administration }\end{array}$ & $\begin{array}{l}\text { Mean Glucose Control } \\
\text { Group (III) (士SD) }[\mathrm{mg} / \mathrm{dL}]\end{array}$ & $\begin{array}{l}\text { Mean Glucose Insulin } \\
\text { Group (I) ( } \pm \mathrm{SD}) \text { [mg/dL] }\end{array}$ & $\begin{array}{l}\text { Mean Glucose Metformin } \\
\text { Group (II) ( } \pm \mathrm{SD})[\mathrm{mg} / \mathrm{dL}]\end{array}$ & $\begin{array}{l}\text { P values for } \\
\text { the ANOVA } \\
\text { test }\end{array}$ \\
\hline $\begin{array}{l}\text { I3 (first day of drug } \\
\text { administration) }\end{array}$ & $389.58 \pm 17.25$ & $415.86 \pm 33.78$ & $427.25 \pm 21.08$ & 0.21 \\
\hline 15 & $408.43 \pm 9.66$ & $408 \pm 43.35$ & $424.37 \pm 25.92$ & 0.48 \\
\hline 17 & $388.71 \pm 45.16$ & $380 \pm 98.87$ & $463.87 \pm 50.46$ & 0.051 \\
\hline 19 & $424.29 \pm 52.55$ & $431.57 \pm 49.09$ & $454.12 \pm 60.93$ & 0.55 \\
\hline 21 & $409 \pm 60.50$ & $377.57 \pm 76.39$ & $448.25 \pm 29.38$ & 0.08 \\
\hline 23 & $371.43 \pm 38.55$ & $413 \pm 71.1$ & $433.5 \pm 33.38$ & 0.075 \\
\hline 25 & $397.39 \pm 52.93$ & $4|2.7| \pm 90.96$ & $439 \pm 35.50$ & 0.44 \\
\hline
\end{tabular}

Note: Statistical significance considered for $p<0.05$

Analysis of the biopsy material collected on day 9 after the formation of the experimental wound in the control group showed massive infiltrates of granulocytes and macrophages, covering the entire volume of the preparations evaluated. In samples derived from wound biopsies of animals from the insulin group, only a small infiltration of inflammatory cells was observed, occurring mainly in the immediate vicinity of the margins of the healing wound, whereas in rats treated with metformin (group II), the inflammatory infiltration was markedly greater than in sections of the insulin (group I) and control (group III) groups. This observation was consistent with the clinical picture evaluated on the basis of photographs (Figure 3).

Preliminary immunohistochemical assessment showed differences in the number of proliferative cell clusters, as evidenced by the increased proportion of Ki67 antigen

INSULIN

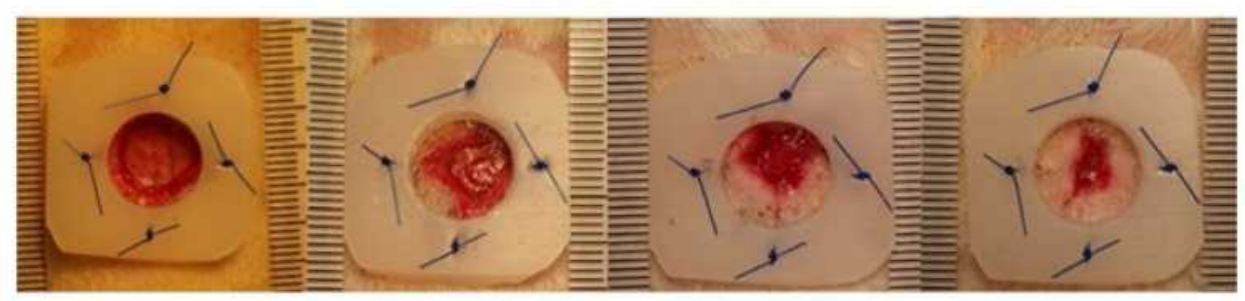

METFORMIN

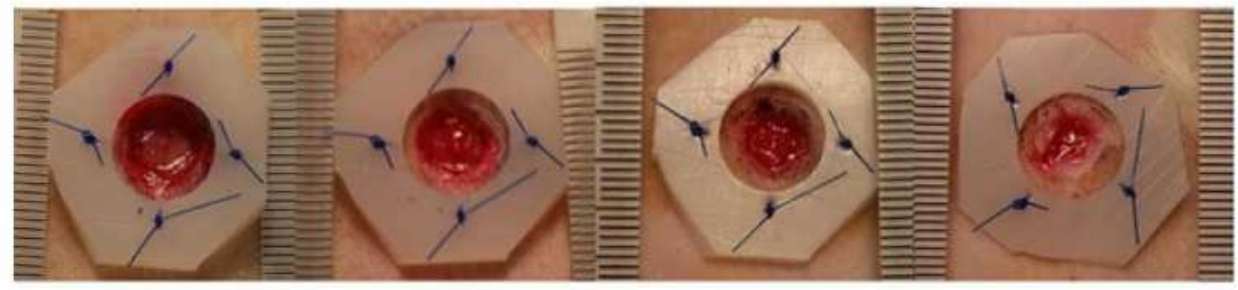

CONTROL

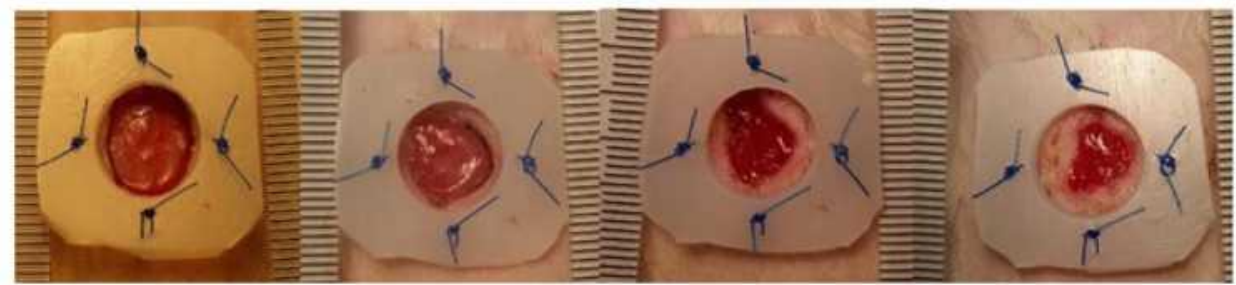

Day 0

Day 3

Day 6

Day 9

Figure 3 Changes in wound surface during observation. 
A
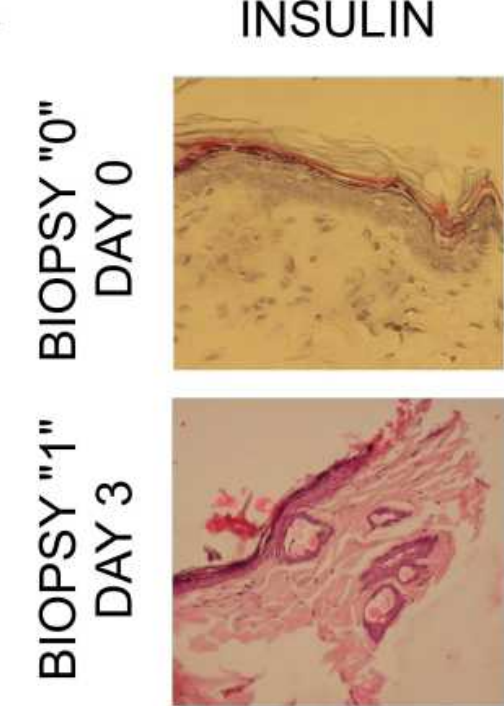

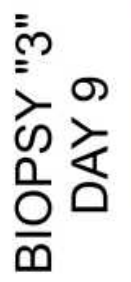

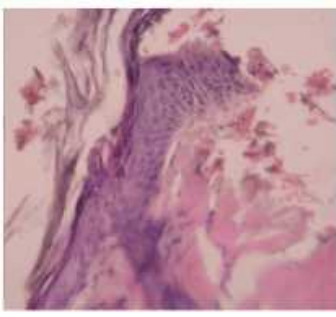

METFORMIN
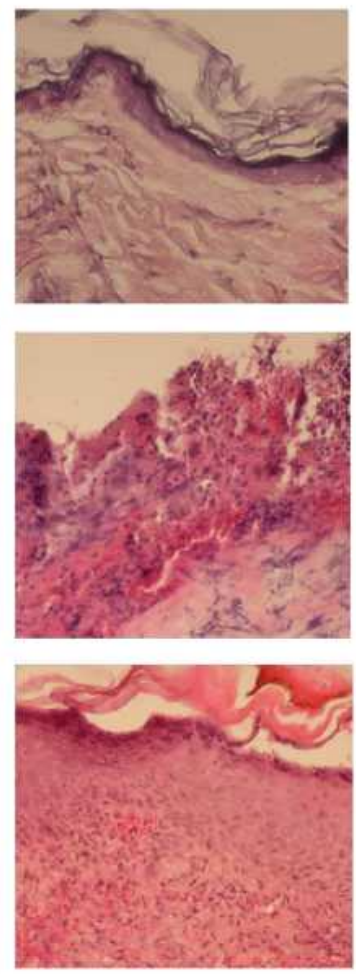

\section{CONTROL}
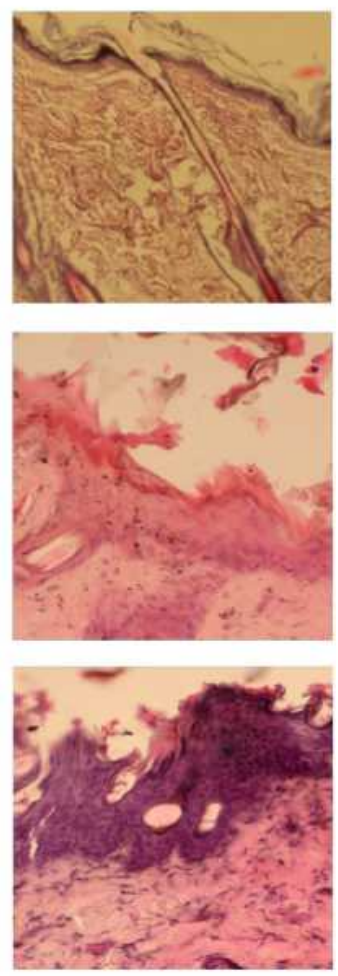

B

\section{Day 3}

\section{Day 9}

Inflammatory infiltrate

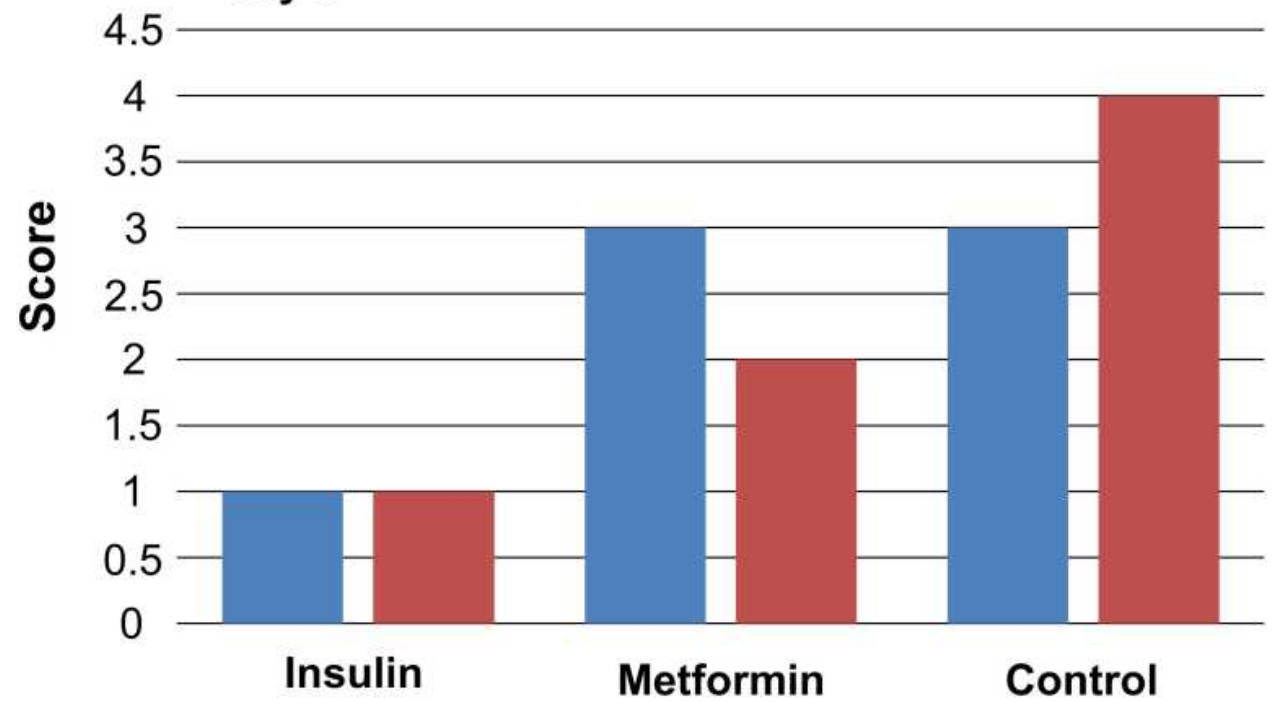

Figure 4 (A) Micrographs of selected rat wounds from groups I (insulin), II (metformin), and III (control), on days 0, 3, and 9 after wound formation. (B) Inflammatory infiltrate (statistical significance not calculated, owing to semiquantitative histological assessment): 0 , no infiltrate; I, weak infiltrate $(<20 \%$ cells in assessed field revealing polymorphonuclear or mononuclear phenotype); 2 , mild infiltrate ( $20 \%-39 \%$ cells revealing polymorphonuclear or mononuclear phenotype); 3 , moderate infiltrate ( $40 \%-$ $59 \%$ cells in assessed field revealing polymorphonuclear or mononuclear phenotype); 4 , intense infiltrate with mild necrosis $(60 \%-80 \%$ cells in assessed field revealing polymorphonuclear or mononuclear phenotype); 5 , severe infiltrate with moderate or extensive necrosis (>80\% cells in assessed field revealing polymorphonuclear or mononuclear phenotype). 
staining in the insulin group (group I) compared with the metformin group (group II) (Figure 5).

\section{Discussion}

Until recently, it has been thought that the presence of a chronic wound in a diabetic patient is an absolute indication for insulin treatment and withdrawal of oral antidiabetic drugs. The experience of the last decade has shown that it is possible to treat this group of patients with oral antidiabetic drugs. However, this approach concentrates on glycemia normalization. The question is whether the wound healing effect in diabetic patients with chronic wounds depends only on glycemia normalization or whether it depends on additional molecular pathways regulated by insulin or other antidiabetic agents.

In our study, we evaluated the effect of selected diabetes medications on the wound healing process in a rat model of streptozotocin-induced diabetes accompanied by neuropathy and chronic hyperglycemia. It is necessary to emphasize that such a study depends on an optimal model of wound healing. In this study, we selected a model that aims to reflect the conditions of the analogous process of diabetic wound healing in human beings as closely as possible. Therefore, a chronic wound model developed during a pilot study was used. ${ }^{25}$ The duration of hyperglycemia in rats in our study may reflect the long-term uncompensated course of diabetes in human beings.

The results of pain threshold measurements confirm the onset of developing sensory neuropathy in the studied rats as early as day 7 after the administration of streptozotocin (Table 1). In human beings, the process of neuropathy development is slower, and, in addition to the existence of diabetes per se, is influenced by such factors as age, glycemic control, elevated levels of cholesterol and triglycerides, hypertension, obesity, and smoking. A study assessing the presence of diabetes complications, EURODIAB IDDM, ${ }^{26}$ which examined more than 3000 patients treated with insulin from 16 countries, initially
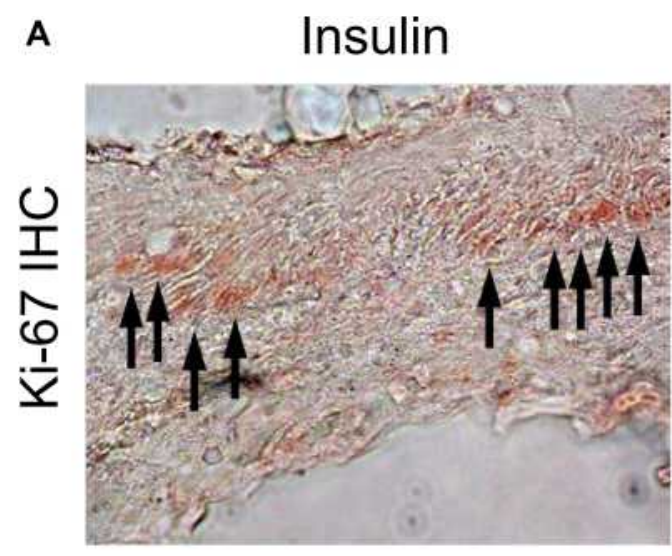

Metformin

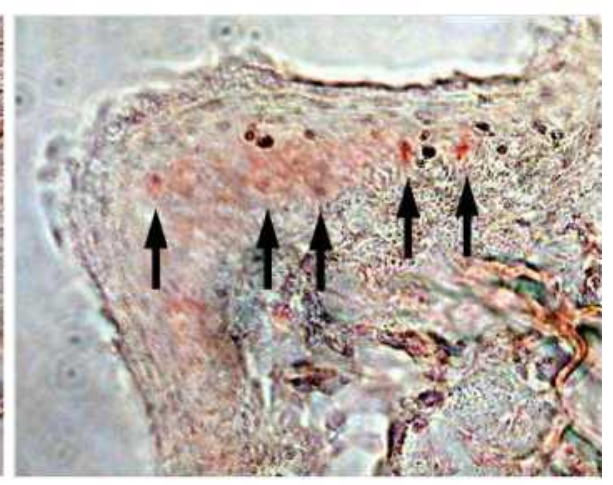

B

Ki-67 positive nuclei

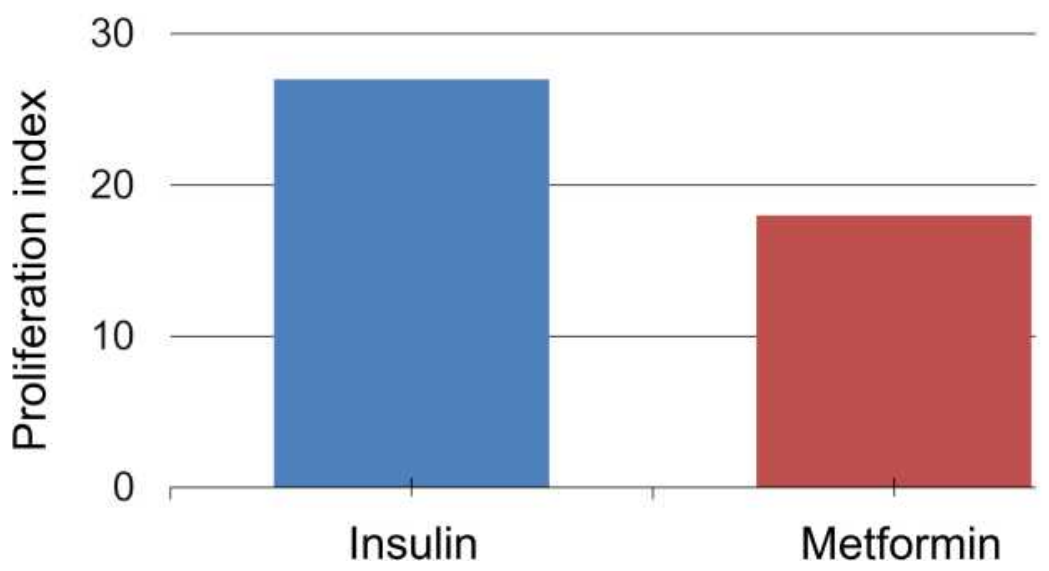

Figure 5 (A) Immunohistochemical assessment with Ki-67 antigen staining in the insulin- and metformin-treated groups (black arrows indicate proliferation centers saturated with Ki-67). (B) Proliferation index, expressed as the percentage of Ki-67-positive nuclei among basal and suprabasal epithelial cells, visualized through immunohistochemical staining (statistical significance not calculated, owing to semiquantitative histological assessment). 
found neuropathy in $28 \%$ of patients; after 7 years of observation, an increase to $51.5 \%$ was recorded. Therefore, our observations based on a rat model are able to provide answers to important clinical questions, because it is possible to reproduce similar conditions in few weeks, while, in the case of a human model, researchers would need many years. Bujalska et al used such a methodology to evaluate the process of developing diabetic neuropathy. ${ }^{27}$

Another issue is wound generation. Most current animal models are based on the formation of acute wounds, which are then subjected to factors responsible for their conversion into chronic wounds, for example, through the development of ischemia (eg, damage associated with pressure) or diabetes. In our study, we present a model imitating the conditions of a chronic wound in patients with diabetes. The inflammation phase is lengthened, the concentration of growth factors and proliferative phase cells is reduced, and neovascularization is impaired. ${ }^{28}$ Thanks to the procedures applied, such as maintaining the state of hyperglycemia for a long time, the use of bacterial lipopolysaccharides, and inhibition of the participation of the panniculus carnosus muscle in the species-specific process of rapid wound healing after injury (by silicone plate placement), it was possible to reflect the conditions of the wound environment observed in a patient with long-term uncontrolled diabetes in the best possible manner. Without the aforementioned modifications, the healing time would have been much shorter ( 5 to 7 days) than was the case in this study, and could not accurately reflect the healing conditions of a chronic wound. ${ }^{29}$

We have selected a rat model with streptozotocininduced diabetes. Although it is not a strict model of type 2 diabetes, we chose it because the evaluation of oral antidiabetic drugs in an animal model of streptozotocin-induced diabetes has become increasingly common practice. $^{30}$ This is partly due to economic aspects but is primarily because it enables the long-term complications of diabetes to be observed within a short time of its induction with streptozotocin. Moreover, it is worth mentioning that oral medications (eg, dapagliflozin) are already approved for the treatment of special cases of type 1 diabetes and a number of studies have been conducted with the use of biguanides (metformin) in this group of patients. ${ }^{31-33}$ Therefore, this model can also be applied to studies of type 2 diabetes. ${ }^{34}$

Insulin and metformin were chosen for assessment as they are the most commonly used drugs in the treatment of diabetes in human beings. The effect of each drug was assessed both by evaluating the percentage of wound surface area reduction, using a novel measuring device, and by immunohistochemical assessment of changes in the expression of $\mathrm{Ki} 67$ antigen in the studied biopsy specimens. ${ }^{35}$ Analysis of variance revealed a significant influence of the treatment method (insulin, metformin, or control) on the relative change in wound area. In our study, it was shown that the wound healing rate was faster in rats receiving insulin than in rats receiving metformin or saline. The rate of wound healing in rats is significantly different from that in human beings; thus, the individual wound healing phases occur at much shorter time intervals. $^{36}$ For this reason, the changes observed over the course of just 3 days of wound treatment in rats may indicate significant differences in the effectiveness of the tested molecule. It was shown that insulin was more efficient than metformin or control treatment in wound area reduction, without any observable differences between metformin and control treatment at any stage. In addition, differences in the concentration of staining of proliferative cell clusters were also demonstrated, as evidenced by the increased Ki67 staining in the group of rats receiving insulin compared with the group receiving metformin and the control group, while maintaining analogous glucose levels. Many authors use the assessment of Ki-67 reactivity to confirm the effectiveness of drug molecules or to show the mechanism of repair processes. ${ }^{12,37}$

Thus, our results demonstrate that insulin treatment tends to be more efficient in wound healing in our diabetic model than metformin, irrespective of the effect of glycemic normalization. This could indicate that insulin and metformin trigger different molecular pathways that influence wound healing in different ways. Although we did not investigate certain molecular pathways in our study, other authors provide insight into that process.

A number of basic studies performed on other animal models (Drosophila melanogaster and Caenorhabditis elegans) provide arguments that insulin and insulin-like growth factor 1 (IGF-1) mediate activation of the PI3K/ Akt signaling pathway, stimulating epithelial cell migration and wound healing. Moreover, Kakanj et al showed that the expression of a dominant-negative version of the insulin receptor in D. melanogaster results in decreased wound healing is associated with the inhibition of translocation of the forkhead box O (FOXO) transcriptional factor from the nucleus to the cytoplasm. ${ }^{38}$ It should also be emphasized that insulin directly stimulates the 
proliferation of keratinocytes through the PI3K/Akt pathway and activates their migration through the activation of Rac1 GTPase, enhancing the entire process of wound healing. ${ }^{39,40}$ Recently, the beneficial effect of topical insulin on wounds has also been observed. ${ }^{41}$ The increase in levels of glucose transporter 1 (GLUT1) protein and its translocation from the cytosol to the cell membrane of the basal epidermal cells demonstrate that diabetic skin tissue is characterized by insulin responsiveness. ${ }^{42}$

By contrast, metformin activates different molecular pathways, which might play a detrimental role in wound healing. Lee et al suggested that metformin inhibits the mTOR pathway, promoting apoptosis, as demonstrated in a study on bile duct cancer cells. ${ }^{29}$ Metformin has been shown to be antiproliferative in a number of studies demonstrating its additional antitumor effect. ${ }^{43}$ Such an antiproliferative effect, although desired in antitumor therapy, is not beneficial in wound healing treatment. This mechanism might explain why metformin therapy, which normalizes glycemia, does not provide any beneficial effect on wound healing, compared with the control group in our study.

There is no unambiguous consensus between researchers on the effect of metformin on wound healing, and the results of studies published so far are contradictory. Verma et al showed, in an in vivo model of mice, that metformin affects the oxidation, metabolism, and proliferation of head and neck squamous cell carcinoma cells. ${ }^{44}$ After 5 days, subjects receiving metformin had a significant increase $(p<0.05)$ in tumor oxygen saturation and hemoglobin concentration levels in treated tumors compared with untreated control subjects, with fluorescence imaging revealing a $46 \%$ decrease in 2-deoxy-D-glucose uptake compared with control subjects. In addition, a statistically significant reduction of Ki-67 antigen staining clusters was found, while smaller tumor sizes were observed using magnetic resonance imaging. ${ }^{44}$ As far as the tumorigenic process is concerned, this appears to be beneficial, whereas a stimulating effect on cell proliferation would be more desirable for wound healing. Different results showing the effect of metformin on wound healing also result from the method of administration (orally or topically, directly to the wound). Qing et al suggest a positive effect of topically applied metformin on wound healing through inhibition of the AMP-activated protein kinase (AMPK) pathway, which leads to macrophage differentiation toward the M2 population. ${ }^{45}$ However, Bagheri et $\mathrm{al}^{46}$ who assessed the effect of metformin and photomodulation on wound healing in rats, confirmed the positive effect of metformin on the differentiation of macrophages in the M2 population, but did not find a positive relationship between the number of M2 macrophages and wound strength. Bagheri et al explain this by pointing to the inhibitory effect of photomodulation on the development of the M2 macrophage population. $^{46}$

Owing to the aforementioned methodologic approach, we were able to conduct a study using an animal model that reflects the conditions of diabetic wound healing in human beings in many aspects. The innovative element of this study is the comparison of the effects of insulin and metformin under similar conditions-poor glycemic control, which is often observed in clinical settings in patients with long-term diabetes.

Another modification in our study was the assessment of the wound surface area using a photoplanimetric method, implemented using the Planimator app, which allows precise measurement of the ulcer surface area. In our previous pilot study, a thermal imaging camera was used, which appeared to give high sensitivity and specificity. However, although thermal imaging provided information about the current metabolic status of the wound, it did not allow accurate measurement of the wound's surface area. In this study, the Planimator app was used for the first time to assess the wound surface area precisely. Preliminary test data indicate greater accuracy and repeatability of surface area measurements made using this application compared with assessments using commercial devices, such as Visitrak $^{\circledR}$ (Smith \& Nephew, Watford, UK) or SilhouetteMobile ${ }^{\circledR}$ (Aranz Medical, Christchurch, New Zealand). ${ }^{25}$ The app was developed at the Institute of Biocybernetics and Biomedical Engineering of the Polish Academy of Sciences (Warsaw, Poland), with the intention of producing, for everyday use by medical personnel, a simple accurate measurement method using two rulers. ${ }^{47}$ The digital photoplanimetric method used in the Planimator app was recently improved, based on a proposed adaptive calibration, which increases measurement accuracy and precision at curved surfaces. ${ }^{48}$

\section{Study Limitations}

Given the complexity of the processes that occur in chronic wounds in adults, particularly in people with diabetes, there is no single animal model that can perfectly reproduce human conditions. Therefore, our goal was to 
choose the optimal model that would suit our study and that could be further modified, if required.

In our study, we used streptozotocin to destroy beta cells in order to produce diabetes in the study animals. There are many animal models of diabetes, including commercially available genetically modified mice or rats, but owing to the financial limitations of the study, genetically modified animals were not used. Each of the aforementioned models allows some insight into the wound healing process in diabetes; the problem is that there are many different protocols, used by different laboratories, making it difficult to compare models and results. Based on the current literature, it is difficult to determine which of the models described is optimal, owing to the many different output parameters (eg, different animal ages or wound sizes) and the adopted conditions and objectives of each study (eg glycemic ranges).

Another important aspect is the difference in biomechanical conditions used in forming and permanently affecting a specific wound. In DFS, one of the main factors in the formation of wounds is pressure on the sole surface of the foot, which, in combination with sensory disturbances and delayed skin regeneration processes (autonomic neuropathy), initially results in thickening of the stratum corneum (forming a callus), and then the formation of wounds. In our study, wounds imitating those found in human beings were created "artificially". Owing to its location on the animal's dorsum, which allows a consistently sized ulcer to be reproduced, there is no constant pressure on its surface, which is often the cause of long and complicated treatment of similar wounds in human beings. It is worth noting, however, that a basic part of the treatment of ulcers in DFS is the patient's use of pressurerelieving footwear or even an absolute ban on applying pressure on the limb. A situation in which there is a lack of biomechanical pressure on a wound formed on a rat's dorsum can therefore be a good representation of a patient's compliance with recommendations for pressure relief.

In the study, metformin and insulin were not used in combined administration in any group; this might have yielded different study results.

In addition, at the beginning of the study, we did not have devices for assessing wounds in $3 \mathrm{D}$ projection, ie for assessing the depths of wounds; such devices are now more easily available.

\section{Conclusion}

Our study provides evidence that insulin accelerates wound healing in a rat model of diabetes with neuropathy and under chronic hyperglycemia, causing increased expression of Ki67 antigen and a reduced wound surface, and that this effect does not depend solely on glycemia normalization. The results obtained in this study may prompt investigations into the broader influence of antidiabetic drugs, including insulin, on the mechanisms of wound healing in diabetes. In the next phases of our study, we will attempt to establish these mechanisms and to explain, molecularly, the noticeable acceleration of wound healing under hyperglycemia in patients treated with insulin.

\section{Abbreviations}

b.m., body mass; AMPK, AMP-activated protein kinase; ANOVA, analysis of variance; DFS, diabetic foot syndrome; FOXO, forkhead box O; NADPH, nicotinamide adenine dinucleotide phosphate; SGLT-2, sodium-glucose co-transporter-2.

\section{Funding}

The study was funded by the statute fund of the Department of Diabetology and Internal Diseases of Medical University of Warsaw for 2016 and the Mini Student Grant of the Medical University of Warsaw for 2015/2016, number 1WO/NM1/2014.

\section{Disclosure}

The authors report no conflicts of interest in this work.

\section{References}

1. Najafian Y, Khorasani ZM, Najafi MN, et al. Efficacy of aloe vera/ plantago major gel in diabetic foot ulcer: a randomized double-blind clinical trial. Curr Drug Discov Technol. 2019;16(2):223-231. doi:10.2174/1570163815666180115093007

2. Armstrong DG, Boulton AJ, Bus SA. Diabetic foot ulcers and their recurrence. $N$ Engl J Med. 2017;376:2367-2375. doi:10.1056/ NEJMra1615439

3. Jeffcoate WJ, Chipchase SY, Ince P, Game FL. Assessing the outcome of the management of diabetic foot ulcers using ulcer-related and person-related measures. Diabetes Care. 2006;29(8):1784-1787. doi: $10.2337 / \mathrm{dc} 06-0306$

4. Prompers L, Schaper N, Apelqvist J, et al. Prediction of outcome in individuals with diabetic foot ulcers: focus on the differences between individuals with and without peripheral arterial disease. The EURODIALE Study. Diabetologia. 2008;51(5):747-755. doi:10.1007/s00125-008-0940-0

5. International Diabetes Federation. Time to Act: Diabetes and Foot Care. Brussels: International Diabetes Federation; 2005.

6. Lu Q, Wang J, Wei X, et al. Cost of diabetic foot ulcer management in China: a 7-year single-center retrospective review. Diabetes Metab Syndr Obes. 2020;13:4249-4260. doi:10.2147/DMSO.S275814

7. Patel DK, Strong J. The pleiotropic effects of sodium-glucose cotransporter-2 inhibitors: beyond the glycemic benefit. Diabetes Ther. 2019;10(5):1771-1792. doi:10.1007/s13300-019-00686-z

8. Christman AL, Selvin E, Margolis DJ, Lazarus GS, Garza LA. Hemoglobin A1c predicts healing rate in diabetic wounds. $J$ Invest Dermatol. 2011;131(10):2121-2127. doi:10.1038/jid.2011.176 
9. Marhoffer W, Stein M, Maeser E, Federlin K. Impairment of polymorphonuclear leukocyte function and metabolic control of diabetes. Diabetes Care. 1992;15(2):256-260. doi:10.2337/diacare.15.2.256

10. Geerlings SE, Hoepelman AI. Immune dysfunction in patients with diabetes mellitus (DM). FEMS Immunol Med Microbiol. 1999;26:259-265. doi:10.1111/j.1574-695X.1999.tb01397.x

11. Witko-Sarsat V, Rieu P, Descamps-Latscha B, et al. Neutrophils: molecules, functions and pathophysiological aspects. Lab Invest. 2000;80:617-653.

12. Hong JP, Park SW. The combined effect of recombinant human epidermal growth factor and erythropoietin on full-thickness wound healing in diabetic rat model. Int Wound J. 2012;11(4):373-378. doi:10.1111/j.1742-481X.2012.01100.x

13. Davidson JM. Animal models for wound repair. Arch Dermatol Res. 1998;290(Suppl 1):S1-S11. doi:10.1007/PL00007448

14. Dorsett-Martin WA. DVM rat models of skin wound healing: a review. Wound Rep Regen. 2004;12:591-599. doi:10.1111/j.10671927.2004.12601.x

15. Small animal anesthesia and analgesia. Polish National Ethical Committee for animal experiments; Good practices. Available from: http://www.bip.nauka.gov.pl/dobre-praktyki/. Accessed May 30, 2019.

16. Nakhoda A, Wong HA. The induction of diabetes in rats by intramuscular administration of streptozotocin. Experientia. 1979;35:1679-1680. doi:10.1007/BF01953269

17. Randall LO, Selitto JJ. A method for measurement of analgesic activity on inflamed tissue. Arch Int Pharmacodyn Ther. 1957;111:409-419.

18. Pinheiro LS, de Melo AD, Andreazzi AE, et al. Protocol of insulin therapy for streptozotocin-diabetic rats based on a study of food ingestion and glycemic variation. Scand J Lab Anim Sci. 2011;38 (2):356-360

19. Ozturk Y, Aydin S. Effects of streptozotocin-induced diabetes and insulin on calcium responsiveness of the rat vas deferens. Life Sci. 2006;78:1084-1090. doi:10.1016/j.1fs.2005.06.019

20. Gottrup F, Agren MS, Karlsmark T. Models for use in wound healing research: a survey focusing on in vitro and in vivo adult soft tissue. Wound Repair Regen. 2000;8:83-96. doi:10.1046/j.1524475x.2000.00083.x

21. Ngawhirunpat T, Hatanaka T, Katayama K, et al. Changes in electrophysiological properties of rat skin with age. Biol Pharm Bull. 2002;25(9):1192-1196. doi:10.1248/bpb.25.1192

22. Babaei S, Bayat M, Nouruzian M, Bayat M. Pentoxifylline improves cutaneous wound healing in streptozotocin-induced diabetic rats. Eur J Pharmacol. 2013;700:165-172. doi:10.1016/j.ejphar.2012.11.024

23. Takimoto K, Kawashima N, Suzuki N, et al. Down-regulation of inflammatory mediator synthesis and infiltration of inflammatory cells by MMP-3 in experimentally induced rat pulpitis. $J$ Endod. 2014;40(9):1404-1409. doi:10.1016/j.joen.2014.04.001

24. Foltynski P. Ways to increase precision and accuracy of wound area measurement using smart devices: advanced app planimator. PLoS One. 2018;13(3):e0192485. doi:10.1371/journal.pone.0192485

25. Mrozikiewicz-Rakowska B, Mieczkowski M, Siwko TJ, et al. Nowy Model Ran Przewlekłych W Cukrzycy - Badanie Pilotażowe U Szczurów Rasy Wistar [New model of chronic wounds in Wistar rats - pilot study]. Leczenie Ran. 2015;12(1):33-39.

26. Tesfaye S, Chaturvedi N, Eaton SEM, et al. Vascular risk factors and diabetic neuropathy. $N$ Engl J Med. 2005;352:341-350. doi:10.1056/ NEJMoa032782

27. Bujalska M, Tatarkiewicz J, de Cordé A, Gumułka SW. Effect of cyclooxygenase and nitric oxide synthase inhibitors on streptozotocin-induced hyperalgesia in rats. Pharmacology. 2008;81 (2):151-157. doi:10.1159/000110787

28. Falanga V. Wound healing and its impairment in the diabetic foot. Lancet. 2005;366(9498):1736-1743. doi:10.1016/S0140-6736(05) 67700-8
29. Lee J, Hong EM, Kim JH, et al. Metformin induces apoptosis and inhibits proliferation through the AMP-activated protein kinase and insulin-like growth factor 1 receptor pathways in the bile duct cancer cells. $J$ Cancer. 2019;10(7):1734-1744. doi:10.7150/jca.26380

30. Gebremeskel L, Beshir Tuem K, Teklu T. Evaluation of antidiabetic effect of ethanolic leaves extract of Becium grandiflorum Lam. (Lamiaceae) in streptozotocin-induced diabetic mice. Diabetes Metab Syndr Obes. 2020;13:1481-1489. doi:10.2147/DMSO. S246996

31. Paik J, Blair HA. Dapagliflozin: a review in type 1 diabetes. Drugs. 2019;79:1877-1884.

32. Petrie JR, Chaturvedi N, Ford I, et al. Cardiovascular and metabolic effects of metformin in patients with type 1 diabetes (REMOVAL): a double-blind, randomised, placebo-controlled trial. Lancet Diabetes Endocrinol. 2017;5(8):597-609. doi:10.1016/S2213-8587(17)30194-8

33. Beysel S, Unsal IO, Kizilgul M, et al. The effects of metformin in type 1 diabetes mellitus. BMC Endocr Disord. 2018;18(1):1. doi:10.1186/s12902-017-0228-9

34. Zhang M, Lv XY, Li J, Xu ZG, Chen L. The characterization of high-fat diet and multiple low-dose streptozotocin induced type 2 diabetes rat model. Exp Diabetes Res. 2008;2008:704045. doi:10.1155/2008/704045

35. Juriková M, Danihel L, Polák S, Varga I. Ki67, PCNA, and MCM proteins: markers of proliferation in the diagnosis of breast cancer. Acta Histochem. 2016;118:544-552. doi:10.1016/j.acthis.201 6.05 .002

36. Galiano RD, Michaels J, Dobryansky M, Levine JP, Gurtner GC. Quantitative and reproducible murine model of excisional wound healing. Wound Repair Regen. 2004;12(4):485-492. doi:10.1111/ j.1067-1927.2004.12404.x

37. Chen Y, Zhang Q, Wu Y, Branch-Brooks CD, Butler CE. Short-term influences of radiation on musculofascial healing in a laparotomy rat model. Sci Rep. 2019;9:11896. doi:10.1038/s41598-019-48201-5

38. Kakanj P, Moussian B, Grönke S, et al. Insulin and TOR signal in parallel through FOXO and S6K to promote epithelial wound healing. Nat Commun. 2016;7:12972. doi:10.1038/ncomms 12972

39. Sabater AL, Andreu EJ, García-Guzmán M, et al. Combined PI3K/ Akt and Smad2 activation promotes corneal endothelial cell proliferation. Invest Ophthalmol Vis Sci. 2017;58(2):745-754. doi:10.1167/iovs.16-20817

40. Hashmi S, Marinkovic MP. Biology of the basement membrane zone. In: Murrell DF, editor. Blistering Diseases: Clinical Features, Pathogenesis, Treatment. Berlin Heidelberg: Springer Verlag; 2015:3-20.

41. Wang J, Xu J. Effects of topical insulin on wound healing: a review of animal and human evidences. Diabetes Metab Syndr Obes. 2020;13:719-727. doi:10.2147/DMSO.S237294

42. Yu T, Gao M, Yang P, et al. Topical insulin accelerates cutaneous wound healing in insulin-resistant diabetic rats. Am J Transl Res. 2017;9(10):4682-4693.

43. Irie H, Banno K, Yanokura M, et al. Metformin: a candidate for the treatment of gynecological tumors based on drug repositioning. Oncol Lett. 2016;11(2):1287-1293. doi:10.3892/ol.2016.4075

44. Verma A, Rich LJ, Vincent-Chong VK, Seshadri M. Visualizing the effects of metformin on tumor growth, vascularity and metabolism in head and neck cancer. J Oral Pathol Med. 2018;47(5):484-491. doi:10.1111/jop.12705

45. Qing L, Fu J, Wu P, et al. Metformin induces the M2 macrophage polarization to accelerate the wound healing via regulating AMPK/ mTOR/NLRP3 inflammasome singling pathway. Am J Transl Res. 2019;11(2):655-668.

46. Bagheri M, Mostafavinia A, Abdollahifar MA, et al. Combined effects of metformin and photobiomodulation improve the proliferation phase of wound healing in type 2 diabetic rats. Biomed Pharmacother. 2020;123:109776. doi:10.1016/j.biopha.2019.109776 
47. Foltynski P, Ladyzynski P, Ciechanowska A, et al. Wound area measurement with digital planimetry: improved accuracy and precision with calibration based on 2 rulers. PLoS One. 2015;10: e0134622. doi:10.1371/journal.pone. 0134622
48. Foltynski P, Ladyzynski P. Digital planimetry with a new adaptive calibration procedure results in accurate and precise wound area measurement at curved surfaces. $J$ Diabetes Sci Technol. 2020:193229682095934. doi:10.1177/1932296820959346

\section{Publish your work in this journal}

Diabetes, Metabolic Syndrome and Obesity: Targets and Therapy is an international, peer-reviewed open-access journal committed to the rapid publication of the latest laboratory and clinical findings in the fields of diabetes, metabolic syndrome and obesity research. Original research, review, case reports, hypothesis formation, expert opinion and commentaries are all considered for publication. The manuscript management system is completely online and includes a very quick and fair peer-review system, which is all easy to use. Visit http://www.dovepress.com/testimonials.php to read real quotes from published authors. 